https://doi.org/10.15407/ujpe65.9.768

M.P. KOZLOVSKII, O.A. DOBUSH

Institute for Condensed Matter Physics, Nat. Acad. of Sci. of Ukraine

(1, Svientsitsky Str., Lviv 79011, Ukraine; e-mail: dobush@icmp.lviv.ua)

\title{
BEHAVIOR OF A BINARY ASYMMETRIC MIXTURE OF INTERACTING PARTICLES IN THE SUPERCRITICAL REGION
}

\begin{abstract}
We propose a method for describing the phase behavior of a system consisting of particles of two sorts. The interaction of each species is described by interaction potentials containing the repulsive and attractive components. Asymmetry is ensured by different values of the interaction potentials of each sort. The grand partition function of a binary mixture is calculated in the zero-mode approximation. A line of critical points, which correspond to different proportions of the components, is calculated for specific values of parameters of the interaction potential. We have obtained an equation that relates the introduced mixing parameter $x$ to the concentration of the system. An explicit expression of the pressure of the binary mixture is derived as a function of the relative temperature and the mixing parameter $x$ to plot the Widom line. It is established that, for boundary values of this parameter $(x=0$ and $x=1)$, the equation of state of a mixture turns into equations of state of its separate species.

Keywords: asymmetric binary mixture, cell fluid model, collective variables, equation of state, Widom line.
\end{abstract}

\section{Introduction}

A crucial part of the theory of phase transitions in multiparticle systems is the elaboration of functional methods. The study of microscopic mechanisms leading to the diversity of the phase behavior in mixtures is relevant for prognosticating the phase diagrams of systems containing particles of different species. The results obtained by both experimental and theoretical methods are essential in this regard. Among the former are the works [1-3] by L.A. Bulavin. We dedicate the present article to the occasion of his 75 th birthday.

Theoretical approaches include publications [4, 5], where the description of multicomponent systems is carried out within the mean-field formalism, and work [6], where the study of a binary system is performed in a more general case. The compilation $[7,8]$ proposes an original approach to explain phase transitions and critical phenomena, which provides an exact functional representation of the grand partition function of a multicomponent model in the method of collective variables [9] with the reference system. There, a hard-sphere system is the reference system, and the

(C) M.P. KOZLOVSKII, O.A. DOBUSH, 2020 interaction potential contains repulsive and attractive parts. The obtained results were generalized to the case of the Coulomb interaction (RPM) model [10]. In [11, 12], the behavior of a binary symmetric mixture was investigated in the framework of that approach near the critical point using non-Gaussian distributions.

More than 60 years ago, experiments on single fluids identified distinct liquid-like and gas-like structures under supercritical conditions [13]. Since then, the interest of the scientific community in the comprehensive study [14-16] of supercritical fluids for their widespread technological applications $[17,18]$ has been steadily increasing due to their high density, solubility, and transport properties. The line between gas-like and liquid-like structures - the Widom line [19] - is an extension to the coexistence curve in the supercritical region, which is characterized by maxima in the thermodynamic response functions. Over the past decade, this crossover line and the properties of supercritical single fluids have been actively investigated [20,21]. Recently, M. Raju et al. have presented an evidence for the existence of Widom lines in binary mixtures from molecular dynamics simulations [22].

ISSN 2071-0194. Ukr. J. Phys. 2020. Vol. 65, No. 9 
The aim of the present article is a description of the phase behavior of a binary fluid in the supercritical region using the cell fluid model $[23,24]$. In contrast to the functional approaches mentioned above, we use a "soft repulsion" as a feature of the reference system. This provides calculating the grand partition function of the model within a single approach of the method of collective variables. The present work summarizes the results of [25] in the case of an asymmetric binary system. We have found that the critical temperature of the mixture is a function of its composition, described a method for calculating the equation of state of the mixture, and shown its pressure behavior along the Widom line.

\section{Calculating the Grand Partition Function of a Binary Asymmetric System}

We use the results of [25] to describe the properties of a binary system of particles. Let the volume $V$ contain $N_{a}$ particles of species $a$ and $N_{b}$ particles of species $b$. The interaction potentials between particles of each sort are modeled as follows:

$U_{\delta}(r)=C_{H}^{(\delta)}\left\{A_{\delta} e^{-n_{0}^{(\delta)}\left(r_{\delta}-R_{0}^{(\delta)}\right) / \alpha_{\delta}}+\right.$
$\left.+e^{-\gamma_{\delta}\left(r_{\delta}-R_{0}^{(\delta)}\right) / \alpha_{\delta}}-2 e^{-\left(r_{\delta}-R_{0}^{(\delta)}\right) / \alpha_{\delta}}\right\}$.

In the latter expression, the index $\delta=a, b$ labels the particle species. For the normalization constants

$C_{H}^{(\delta)}=D \frac{n_{0}^{(\delta)}}{n_{0}^{(\delta)}+\gamma_{\delta}-2} ; \quad A_{\delta}=\frac{2-\gamma_{\delta}}{n_{0}^{(\delta)}}$,

we have the following conditions: 1) the minimum of $U_{\delta}(r)$ is at $\left.r_{\delta}=R_{0}^{(\delta)}, 2\right)$ its absolute value equals the energy of dissociation $-D$. For simplicity, assume that $\alpha_{a}=\alpha_{b}=\alpha$ is the effective interaction radius, and $R_{0}^{(a)}=R_{0}^{(b)}=R_{0}$. Moreover, we use $R_{0}$-units to measure all the distance quantities. The interaction potentials $U_{a}(r)$ and $U_{b}(r)$ differ from each other by the parameters $\gamma_{a} \neq \gamma_{b}$ and $n_{0}^{(a)} \neq n_{0}^{(b)}$. The values of these parameters used in the present research are given in Appendix A. However, they might be changed to reflect other particular systems. The fluid system is henceforth modeled as a cell fluid [26], in which the total volume $V$ of the system is divided into $N_{v}$ cubic cells each of the side $c$ and the volume $v=c^{3}$. The lattice analog of (1) is expressed as

$U_{l_{12}}^{(\delta)}=C_{H}^{(\delta)}\left\{A_{\delta} e^{-\frac{n_{0}^{(\delta)}\left(l_{12}-c\right)}{\alpha_{R} c}}+e^{-\frac{\gamma_{\delta}\left(l_{12}-c\right)}{\alpha R^{c}}}-\right.$

ISSN 2071-0194. Ukr. J. Phys. 2020. Vol. 65, No. 9 $\left.-2 e^{-\frac{l_{12}-c}{\alpha R^{c}}}\right\}$

Here, $\alpha_{R}=\alpha / R_{0}$ is a dimensionless quantity, $l_{12}=\left|\mathbf{l}_{1}-\mathbf{l}_{2}\right|$ is a distance between the cell vectors $\mathbf{l}_{1}, \mathbf{l}_{2} \in \Lambda$ :

$\Lambda=\left\{l=\left(l_{x}, l_{y}, l_{z}\right) \mid l_{i}=c n_{i} ; n_{i}=1,2, \ldots, N_{i} ;\right.$

$\left.i=x, y, z ; N_{i}=\left(N_{v}\right)^{1 / 3}\right\}$.

In the thermodynamic limit $V \rightarrow \infty, N_{v} \rightarrow \infty$, $v=V / N_{v}=$ const. The Fourier transform of (3) has the form

$\Phi_{\delta}(k)=\Psi_{\delta}(k)+\Phi_{\delta}^{(r)}(k)-\Phi_{\delta}^{(a)}(k)$,

where both $\Phi_{\delta}^{(r)}(k)$ and $\Psi_{\delta}(k)$ are the repulsive parts of the interaction between species $\delta$, and $\Phi_{\delta}^{(a)}(k)$ is the attraction. We have

$\Psi_{\delta}(k)=C_{H}^{(\delta)} A_{\delta} 8 \pi e^{\frac{n_{0}^{(\delta)}}{\alpha_{R}}}\left(\frac{\alpha_{R}}{n_{0}^{(\delta)}}\right)^{3}\left[1+\left(\frac{\alpha_{R}}{n_{0}^{(\delta)}} c k\right)^{2}\right]^{-2}$,

$\Phi_{\delta}^{(r)}(k)=C_{H}^{(\delta)} 8 \pi e^{\gamma_{\delta} / \alpha_{R}}\left(\frac{\alpha_{R}}{\gamma_{\delta}}\right)^{3}\left[1+\left(\frac{\alpha_{R}}{\gamma_{\delta}} c k\right)^{2}\right]^{-2}$,

$\Phi_{\delta}^{(a)}(k)=C_{H}^{(\delta)} 16 \pi e^{1 / \alpha_{R}} \alpha_{R}^{3}\left[1+\left(\alpha_{R} c k\right)^{2}\right]^{-2}$.

Recall that $\delta=a, b$. According to the results of [25], we write the expression for the grand partition function of a binary cell fluid model as follows:

$\Xi=g_{V}^{(a)} g_{V}^{(b)} \exp \left[N_{v}\left(a_{0}-\frac{\beta \tilde{\mu}_{a}^{2}}{2 W_{a}(0)}\right)\right] \times$
$\times \exp \left[N_{v}\left(b_{0}-\frac{\beta \tilde{\mu}_{b}^{2}}{2 W_{b}(0)}\right)\right] \int\left(d t_{\mathbf{k}}^{(a)}\right)^{N_{v}} \times$
$\times \exp \left[N_{v}^{\frac{1}{2}}\left(\frac{\tilde{\mu}_{a}}{W_{a}(0)}+a_{1}\right) t_{0}^{(a)}+\frac{1}{2} \sum_{\mathbf{k} \in \mathcal{B}_{\mathrm{c}}} D_{a}(k) t_{\mathbf{k}}^{(a)} t_{-\mathbf{k}}^{(a)}+\right.$
$\left.+\sum_{n=3}^{\infty} \frac{a_{n}}{n !} N_{v}^{\frac{2-n}{n}} \sum_{\mathbf{k}_{1}, \ldots, \mathbf{k}_{n}} t_{\mathbf{k}_{1}}^{(a)} \ldots t_{\mathbf{k}_{n}}^{(a)} \delta_{\mathbf{k}_{1}+\ldots+\mathbf{k}_{n}}\right] \times$
$\times \int\left(d t_{\mathbf{k}}^{(b)}\right)^{N_{v}} \exp \left[N_{v}^{\frac{1}{2}}\left(\frac{\tilde{\mu}_{b}}{W_{b}(0)}+b_{1}\right) t_{0}^{(b)}+\right.$
$+\frac{1}{2} \sum_{\mathbf{k} \in \mathcal{B}_{\mathrm{c}}} D_{b}(k) t_{\mathbf{k}}^{(b)} t_{-\mathbf{k}}^{(b)}+\sum_{n=3}^{\infty} \frac{b_{n}}{n !} N_{v}^{\frac{2-n}{n}} \times$
$\left.\times \sum_{\mathbf{k}_{1}, \ldots, \mathbf{k}_{n}} t_{\mathbf{k}_{i}}^{(b)} \ldots t_{\mathbf{k}_{n}}^{(b)} \delta_{\mathbf{k}_{1}+\ldots+\mathbf{k}_{n}}\right]$. 
Here, $\tilde{\mu}_{\delta}=\mu_{\delta}-\mu_{\delta}^{*} \beta_{\mathrm{c}}^{(\delta)} / \beta, \mu_{\delta}^{*}$ is a constant, and $\beta_{\mathrm{c}}^{(\delta)}$ is the inverse critical temperature of species $\delta$ $(\delta=a, b)$. The effective potentials of interaction [25] read

$$
\begin{aligned}
& W_{\delta}(k)=\Phi_{\delta}^{(a)}(k)-\Phi_{\delta}^{(r)}(k)-\Psi_{\delta}(k)+\frac{\beta_{\mathrm{c}}^{(\delta)}}{\beta} \Psi_{\delta}(0), \\
& D_{a}(k)=a_{2}-\frac{1}{\beta W_{a}(k)}, \quad D_{b}(k)=b_{2}-\frac{1}{\beta W_{b}(k)} . \\
& g_{V}^{(\delta)}=\prod_{\mathbf{k} \in \mathcal{B}_{\mathrm{c}}}\left(2 \pi \beta W_{\delta}(k)\right)^{-1 / 2} .
\end{aligned}
$$

The cumulants $a_{n}$ are as follows:

$$
\begin{aligned}
a_{0} & =\ln T_{0}\left(\alpha_{a}^{*}, p_{a}\right), \\
a_{1} & =\frac{T_{1}\left(\alpha_{a}^{*}, p_{a}\right)}{T_{1}\left(\alpha_{a}^{*}, p_{a}\right)}, \\
a_{2} & =\frac{T_{2}\left(\alpha_{a}^{*}, p_{a}\right)}{T_{1}\left(\alpha_{a}^{*}, p_{a}\right)}-a_{1}^{2}, \\
a_{3} & =\frac{T_{3}\left(\alpha_{a}^{*}, p_{a}\right)}{T_{1}\left(\alpha_{a}^{*}, p_{a}\right)}-a_{1}^{3}+3 a_{1} a_{2}, \\
a_{4} & =\frac{T_{4}\left(\alpha_{a}^{*}, p_{a}\right)}{T_{1}\left(\alpha_{a}^{*}, p_{a}\right)}-a_{1}^{4}-6 a_{1}^{2} a_{2}-4 a_{1} a_{3}-3 a_{2}^{2},
\end{aligned}
$$

where the special functions $T_{n}\left(\alpha_{a}^{*}, p_{a}\right)$ read

$T_{n}\left(\alpha_{a}^{*}, p_{a}\right)=\sum_{m=0}^{\infty} \frac{\left(\alpha_{a}^{*}\right)^{m}}{m !} m^{n} e^{-p_{a} m^{2}}$.

Here, $\alpha_{a}^{*}=v \exp \left[\beta_{\mathrm{c}}^{(a)} \mu_{a}^{*}\right]$, and the parameter $p_{a}$ is expressed via the quantity $\Psi_{a}(0)$ :

$p_{a}=\frac{1}{2} \beta_{\mathrm{c}}^{(a)} \Psi_{a}(0)$.

So, it depends on the parameters $n_{0}^{(a)}$ and $\gamma_{a}$ of the interaction potential $U_{l_{12}}^{(a)}$ given by $(3)$.

The cumulants $b_{n}$ are expressed by the formulas similar to (9), where the special functions $S_{n}\left(\alpha_{b}^{*}, p_{b}\right)$

$S_{n}\left(\alpha_{b}^{*}, p_{b}\right)=\sum_{m=0}^{\infty} \frac{\left(\alpha_{b}^{*}\right)^{m}}{m !} m^{n} e^{-p_{b} m^{2}}$

are substituted for the functions $T_{n}\left(\alpha_{a}^{*}, p_{a}\right), \alpha_{b}^{*}=$ $=v \exp \left[\beta_{\mathrm{c}}^{(b)} \mu_{b}^{*}\right]$, and $p_{b}$ is as follows:

$p_{b}=\frac{1}{2} \beta_{c}^{(b)} \Psi_{b}(0)$.
At the present stage, expression (7) for the grand partition function does not contain any approximations, but has infinite series in the exponent. To deal with this case, we use the traditional approximation for the phase transition theory, assuming that $a_{n}$ and $b_{n}$ with $n \geq 5$ are zero. In general, the calculation scheme below allows us to account for the higher powers of the variable $\rho_{\mathbf{k}}^{(\delta)}$, as we did in [27] when investigating the second-order phase transition for the Ising model.

So, we restrict the problem to the approximation of the $\rho^{4}$-model, make the change of variables to eliminate the third power of variables $\rho_{\mathbf{k}}^{(\delta)}$, and get

$$
\begin{aligned}
& \Xi=g_{v}^{(a)} g_{v}^{(b)} \int\left(d \rho^{(a)}\right)^{N_{v}} e^{N_{v} E_{\mu}^{(a)}} \exp \left[E\left(M_{a}, \rho_{\mathbf{k}}^{(a)}\right)\right] \times \\
& \times \int\left(d \rho^{(b)}\right)^{N_{v}} e^{N_{v} E_{\mu}^{(b)}} \exp \left[E\left(M_{b}, \rho_{\mathbf{k}}^{(b)}\right)\right] .
\end{aligned}
$$

For $E_{\mu}^{(\delta)}$, we have

$$
\begin{aligned}
& E_{\mu}^{(a)}=a_{0}-\frac{\beta \tilde{\mu}_{a}^{2}}{2 W_{a}(0)}+m_{a}\left(a_{1}+\frac{\tilde{\mu}_{a}}{W_{a}(0)}\right)+ \\
& +\frac{1}{2} m_{a}^{2} d_{a}(0)+\frac{a_{3}^{4}}{8 a_{4}^{3}}, \\
& E_{\mu}^{(b)}=b_{0}-\frac{\beta \tilde{\mu}_{b}^{2}}{2 W_{b}(0)}+m_{b}\left(b_{1}+\frac{\tilde{\mu}_{b}}{W_{b}(0)}\right)+ \\
& +\frac{1}{2} m_{b}^{2} d_{b}(0)+\frac{b_{3}^{4}}{8 b_{4}^{3}}, \\
& \frac{\tilde{\mu}_{a}}{W_{a}(0)}=M_{a}-a_{1}-m_{a} d_{a}(0)+\frac{a_{3}^{3}}{a_{4}^{2}} \\
& \frac{\tilde{\mu}_{b}}{W_{b}(0)}=M_{b}-b_{1}-m_{b} d_{b}(0)+\frac{b_{3}^{3}}{b_{4}^{2}}
\end{aligned}
$$

here $m_{a}=-\frac{a_{3}}{a_{4}}, m_{b}=-\frac{b_{3}}{b_{4}}$. The functions $E\left(M_{\delta}, \rho_{\mathbf{k}}^{(\delta)}\right)$ are given by

$$
\begin{aligned}
& E\left(M_{a}, \rho_{\mathbf{k}}^{(a)}\right)=N_{v}^{\frac{1}{2}} M_{a} \rho_{0}^{(a)}+\frac{1}{2} \sum_{\mathbf{k} \in \mathcal{B}_{\mathrm{c}}} d_{a}(k) \rho_{\mathbf{k}}^{(a)} \rho_{-\mathbf{k}}^{(a)}+ \\
& +\frac{a_{4}}{24} \frac{1}{N_{v}} \sum_{\substack{\mathbf{k}_{1}, \ldots, \mathbf{k}_{4} \\
\mathbf{k}_{i} \in \mathcal{B}_{\mathrm{c}}}} \rho_{\mathbf{k}_{1}}^{(a)} \ldots \rho_{\mathbf{k}_{4}}^{(a)} \delta_{\mathbf{k}_{1} \ldots \mathbf{k}_{4}}, \\
& E\left(M_{b}, \rho_{\mathbf{k}}^{(b)}\right)=N_{v}^{\frac{1}{2}} M_{b} \rho_{0}^{(b)}+\frac{1}{2} \sum_{\mathbf{k} \in \mathcal{B}_{\mathrm{c}}} d_{b}(k) \rho_{\mathbf{k}}^{(b)} \rho_{-\mathbf{k}}^{(b)}+ \\
& +\frac{b_{4}}{24} \frac{1}{N_{v}} \sum_{\substack{\mathbf{k}_{1}, \ldots, \mathbf{k}_{4} \\
\mathbf{k}_{i} \in \mathcal{B}_{\mathrm{c}}}} \rho_{\mathbf{k}_{1}}^{(b)} \ldots \rho_{\mathbf{k}_{4}}^{(b)} \delta_{\mathbf{k}_{1} \ldots \mathbf{k}_{4} .} .
\end{aligned}
$$

ISSN 2071-0194. Ukr. J. Phys. 2020. Vol. 65, No. 9 
The quantities $d_{\delta}(k)$ are expressed by

$$
\begin{aligned}
& d_{a}(k)=\tilde{a}_{2}-\frac{1}{\beta W_{a}(k)}, \quad \tilde{a}_{2}=a_{2}-\frac{a_{3}^{2}}{2 a_{4}}, \\
& d_{b}(k)=\tilde{b}_{2}-\frac{1}{\beta W_{b}(k)}, \quad \tilde{b}_{2}=b_{2}-\frac{b_{3}^{2}}{2 b_{4}} .
\end{aligned}
$$

According to (6), we have

$$
\begin{aligned}
& \Phi_{\delta}^{(a)}(0)=B_{\delta} \Phi_{\delta}^{(r)}(0), \\
& \Psi_{\delta}^{(a)}(0)=A_{\gamma}^{(\delta)} \Phi_{\delta}^{(r)}(0), \\
& B_{\delta}=2 \gamma_{\delta}^{3} \exp \left[\frac{1-\gamma_{\delta}}{\alpha_{R}}\right], \\
& A_{\gamma}^{(\delta)}=A_{\delta}\left(\frac{\gamma_{\delta}}{n_{0}^{(\delta)}}\right)^{3} \exp \left[\frac{n_{0}^{(\delta)}-\gamma_{\delta}}{\alpha_{R}}\right] .
\end{aligned}
$$

So, we can rewrite $p_{a}$ and $p_{b}$ given by (12) and (14) as follows:

$p_{a}=\frac{1}{2} \beta_{\mathrm{c}}^{(a)} \Phi_{a}^{(r)}(0) A_{\gamma}^{(a)}, \quad p_{b}=\frac{1}{2} \beta_{\mathrm{c}}^{(b)} \Phi_{b}^{(r)}(0) A_{\gamma}^{(b)}$,

and

$$
\begin{aligned}
& W_{a}(0)=\Phi_{a}^{(r)}(0)\left[B_{a}-1+\tau_{a} A_{\gamma}^{(a)}\right], \\
& W_{b}(0)=\Phi_{b}^{(r)}(0)\left[B_{b}-1+\tau_{b} A_{\gamma}^{(b)}\right] .
\end{aligned}
$$

The quantites $\tau_{a}$ and $\tau_{b}$ are the relative temperatures of pure components of the mixture (separately for species $a$ and $b$, respectively):

$\tau_{a}=\frac{T-T_{\mathrm{c}}^{(a)}}{T_{\mathrm{c}}^{(a)}}, \quad \tau_{b}=\frac{T-T_{\mathrm{c}}^{(b)}}{T_{\mathrm{c}}^{(b)}}$.

Obviously, the critical temperature of the mixture $T_{\mathrm{c}}$ is different from $T_{\mathrm{c}}^{(\delta)}$. Taking that into account, we write the following:

$$
\begin{aligned}
\tau_{a} & =\frac{\tau T_{\mathrm{c}}}{T_{\mathrm{c}}^{(a)}}+\frac{T_{\mathrm{c}}-T_{\mathrm{c}}^{(a)}}{T_{\mathrm{c}}^{(a)}}, \\
\tau_{b} & =\frac{\tau T_{\mathrm{c}}}{T_{\mathrm{c}}^{(b)}}+\frac{T_{\mathrm{c}}-T_{\mathrm{c}}^{(b)}}{T_{\mathrm{c}}^{(b)}},
\end{aligned}
$$

where $\tau$ is the relative temperature of the mixture:

$\tau=\frac{T-T_{\mathrm{c}}}{T_{\mathrm{c}}}$.

ISSN 2071-0194. Ukr. J. Phys. 2020. Vol. 65, No. 9
From (22) and (24), we obtain

$$
\begin{aligned}
& W_{a}(0)=\Phi_{a}^{(r)}(0)\left[B_{a}-1+\tau A_{\gamma}^{(a)} \frac{T_{\mathrm{c}}}{T_{\mathrm{c}}^{(a)}}+\frac{T_{\mathrm{c}}-T_{\mathrm{c}}^{(a)}}{T_{\mathrm{c}}^{(a)}} A_{\gamma}^{(a)}\right], \\
& W_{b}(0)=\Phi_{b}^{(r)}(0)\left[B_{b}-1+\tau A_{\gamma}^{(b)} \frac{T_{\mathrm{c}}}{T_{\mathrm{c}}^{(b)}}+\frac{T_{\mathrm{c}}-T_{\mathrm{c}}^{(b)}}{T_{\mathrm{c}}^{(b)}} A_{\gamma}^{(b)}\right] .
\end{aligned}
$$

In the boundary case, either $T_{\mathrm{c}}=T_{\mathrm{c}}^{(a)}$ or $T_{\mathrm{c}}=T_{\mathrm{c}}^{(b)}$, the expressions given by (25) transform into (21). The quantities $T_{\mathrm{c}}^{(\delta)}$ are defined from the conditions $d_{\delta}(0)=0$ and are equal to

$$
\begin{aligned}
& k_{\mathrm{B}} T_{\mathrm{c}}^{(a)}=\tilde{a}_{2} W_{a}\left(0, T_{\mathrm{c}}^{(a)}\right)=\tilde{a}_{2} \Phi_{a}^{(r)}(0)\left(B_{a}-1\right), \\
& k_{\mathrm{B}} T_{\mathrm{c}}^{(b)}=\tilde{b}_{2} W_{b}\left(0, T_{\mathrm{c}}^{(b)}\right)=\tilde{b}_{2} \Phi_{b}^{(r)}(0)\left(B_{b}-1\right) .
\end{aligned}
$$

Note that, because of (21), we can use the equalities

$$
\begin{aligned}
& \Phi_{a}^{(r)}(0) A_{\gamma}^{(a)}=2 p_{a} k_{\mathrm{B}} T_{\mathrm{c}}^{(a)} \\
& \Phi_{b}^{(r)}(0) A_{\gamma}^{(b)}=2 p_{b} k_{\mathrm{B}} T_{\mathrm{c}}^{(b)}
\end{aligned}
$$

to find the critical temperature of the mixture.

\section{The Zero-Mode Approximation}

Recently in [25], we have shown that the zero-mode approximation describes well the phase behavior of a one-component fluid. It is a mean-field-type approximation considering the contribution from the variable $\rho_{\mathbf{k}}$ at $\mathbf{k}=0$. The contributions from $\rho_{\mathbf{k}}$ at $\mathbf{k} \neq 0$ are important near the critical point. Let us investigate the role of $\rho_{0}^{(\delta)}$ in the formation of a phase diagram of the mixture.

The grand partition function of the mixture in the zero-mode approximation is as follows:

$\Xi_{0}=g_{V}^{(a)} g_{V}^{(b)} \exp \left[N_{v}\left(E_{\mu}^{(a)}+E_{\mu}^{(b)}+E_{a}\left(\bar{\rho}_{a}\right)+E_{b}\left(\bar{\rho}_{b}\right)\right)\right]$

The expressions of $E_{\mu}^{(\delta)}$ are given in (16). For $E_{0}\left(\bar{\rho}_{\delta}\right)$, we have

$$
\begin{aligned}
& E_{a}\left(\bar{\rho}_{a}\right)=M_{a} \bar{\rho}_{a}+\frac{1}{2} d_{a}(0) \bar{\rho}_{a}^{2}+\frac{a_{4}}{24} \bar{\rho}_{a}^{4} \\
& E_{b}\left(\bar{\rho}_{b}\right)=M_{b} \bar{\rho}_{b}+\frac{1}{2} d_{b}(0) \bar{\rho}_{b}^{2}+\frac{b_{4}}{24} \bar{\rho}_{b}^{4} .
\end{aligned}
$$

It is convenient to use the Laplace method [28] to calculate (28). Thereby, the values $\bar{\rho}_{a}$ and $\bar{\rho}_{b}$ correspond 
to maxima of the function $E_{0}\left(\bar{\rho}_{a}\right)$ and $E_{0}\left(\bar{\rho}_{b}\right)$, respectively. So, the following equations determine $\bar{\rho}_{a}$ and $\bar{\rho}_{b}$ :

$$
\begin{aligned}
& M_{a}+d_{a}(0) \bar{\rho}_{a}+\frac{a_{4}}{6} \bar{\rho}_{a}^{3}=0, \\
& M_{b}+d_{b}(0) \bar{\rho}_{b}+\frac{b_{4}}{6} \bar{\rho}_{b}^{3}=0 .
\end{aligned}
$$

Equalities (27) define the critical temperatures of each subsystem, which are calculated in [25]. Expression (28) contains independent contributions from the particles of different species.

Now, we introduce the mixing parameter $x$ as follows:

$\bar{\rho}_{a}=x \rho_{+}, \quad \bar{\rho}_{b}=(1-x) \rho_{+}$.

It is easy to see that

$$
\begin{aligned}
& \rho_{+}=\bar{\rho}_{a}+\bar{\rho}_{b}, \\
& x=\frac{\bar{\rho}_{a}}{\bar{\rho}_{a}+\bar{\rho}_{b}}, \quad 1-x=\frac{\bar{\rho}_{b}}{\bar{\rho}_{a}+\bar{\rho}_{b}} .
\end{aligned}
$$

The mixing parameter $x$ is like the concentration in a different space. In Section 4, we show the relation between the parameter $x$ and the concentration of a solution. In terms of $\rho_{+}$and $x$, expressions (29) have the form

$E_{a}\left(x, \rho_{+}\right)=M_{a} x \rho_{+}+\frac{1}{2} d_{a}(0) x^{2} \rho_{+}^{2}+\frac{a_{4}}{24} x^{4} \rho_{+}^{4}$,

$E_{b}\left(x, \rho_{+}\right)=M_{b}(1-x) \rho_{+}+\frac{1}{2} d_{b}(0)(1-x)^{2} \rho_{+}^{2}+$

$+\frac{b_{4}}{24}(1-x)^{4} \rho_{+}^{4}$.

Their sum (see (28)) is as follows:

$E_{a b}\left(x, \rho_{+}\right)=M_{a b}+\frac{1}{2} D_{a b} \rho_{+}^{2}-a_{+} \rho_{+}^{4}$,

where we use notations

$$
\begin{aligned}
& M_{a b}=x M_{a}+(1-x) M_{b}, \\
& D_{a b}=x^{2} d_{a}(0)+(1-x)^{2} d_{b}(0), \\
& a_{+}=-x^{4} a_{4}-(1-x)^{4} b_{4} .
\end{aligned}
$$

In terms of $\rho_{+}$and $x$, the grand potential of the mixture reads

$\Omega=-k_{\mathrm{B}} T \ln \Xi_{0}\left(x, \rho_{+}\right)$.
In order to minimize it with respect to $\rho_{+}$, we have to solve the equation

$\frac{\partial E_{a b}\left(x, \rho_{+}\right)}{\partial \rho_{+}}=0$

namely,

$M_{a b}=-D_{a b}(x, \tau) \rho_{+}+\frac{a_{+}}{6} \rho_{+}^{3}$.

An equation of this type also holds for a single system. Equation (38) is peculiar for the coefficients $D_{a b}(x, T)$ and $a_{+}(x)$ which are functions of the mixing parameter $x$. Moreover, $D_{a b}$ also depends on the relative temperature of the mixture $\tau$, while the relative temperatures $\tau_{a}$ and $\tau_{b}$ characterize pure species $a$ and $b$, respectively.

The effective chemical potential of the mixture $M_{a b}$ is a variable of the same type as either $M_{a}$ for the subsystem of particles of species $a$, or $M_{b}$ for species $b$. Each of them associate with $\mu_{a}$ and $\mu_{b}$. Using the quantity $M_{a b}$ (or $M_{a}, M_{b}$ ) is convenient, because the first-order phase transition occurs at $M_{a}=0$ for the subsystem of particles of species $a, M_{b}=0$ for the subsystem of particles of species $b$ and $M_{a b}=0$ for the mixture of particles of both species $a$ and $b$.

Both Eqs. (38) and (30) are of the same type. Therefore, the condition

$D_{a b}\left(x, T_{\mathrm{c}}\right)=0$

defines the critical temperature of the liquid-gas phase transition of a binary system. Taking (35) into account, we obtain the equation for $T_{\mathrm{c}}=T_{\mathrm{c}}(x)$ :

$x^{2}\left[\tilde{a}_{2}-\frac{1}{\beta_{\mathrm{c}} W_{a}\left(0, T_{\mathrm{c}}\right)}\right]+(1-x)^{2}\left[\tilde{b}_{2}-\frac{1}{\beta_{\mathrm{c}} W_{b}\left(0, T_{\mathrm{c}}\right)}\right]=0$.

We use (26) and (27) to solve the latter equation and get

$\tilde{a}_{2} W_{a}\left(0, T_{\mathrm{c}}\right)=T_{c a}+2 \tilde{a}_{2} p_{a} T_{\mathrm{c}}$,

$\tilde{b}_{2} W_{b}\left(0, T_{\mathrm{c}}\right)=T_{c b}+2 \tilde{b}_{2} p_{b} T_{\mathrm{c}}$,

where

$$
\begin{aligned}
& T_{c a}=T_{\mathrm{c}}^{(a)}\left(1-2 \tilde{a}_{2} p_{a}\right), \\
& T_{c b}=T_{\mathrm{c}}^{(b)}\left(1-2 \tilde{b}_{2} p_{b}\right) .
\end{aligned}
$$

ISSN 2071-0194. Ukr. J. Phys. 2020. Vol. 65, No. 9 
Then we rewrite (40) as follows:

$æ \tilde{a}_{2} W_{a}\left(0, T_{\mathrm{c}}\right) \tilde{b}_{2} W_{b}\left(0, T_{\mathrm{c}}\right)-x^{2} T_{\mathrm{c}} \tilde{b}_{2} W_{b}\left(0, T_{\mathrm{c}}\right) / \tilde{b}_{2}-$

$-(1-x)^{2} T_{\mathrm{c}} \tilde{a}_{2} W_{a}\left(0, T_{\mathrm{c}}\right) / \tilde{a}_{2}=0$.

Here, expression (40) is multiplied by $W_{a}\left(0, T_{\mathrm{c}}\right) \times$ $\times W_{b}\left(0, T_{\mathrm{c}}\right) \neq 0$ and, for $æ$, reads

$æ=\frac{x^{2}}{\tilde{b}_{2}}+\frac{(1-x)^{2}}{\tilde{a}_{2}}$.

Substituting expressions (41) in formula (43), we obtain the quadratic equation

$f_{2} T_{\mathrm{c}}^{2}+f_{1} T_{\mathrm{c}}+f_{0}=0$

where

$$
\begin{aligned}
& f_{2}=4 æ p_{a} p_{b} \tilde{a}_{2} \tilde{b}_{2}-2 x^{2} p_{b}-2(1-x) p_{a}, \\
& f_{1}=2 æ\left(\tilde{a}_{2} p_{a} T_{c b}+\tilde{b}_{2} p_{b} T_{c a}\right)-x^{2} \frac{T_{c b}}{\tilde{b}_{2}}- \\
& -(1-x)^{2} \frac{T_{c a}}{\tilde{a}_{2}}, \\
& f_{0}=æ T_{c a} T_{c b} .
\end{aligned}
$$

The critical temperature of the mixture as a function of the mixing parameter $x \in[0,1]$ is shown in Fig. 1 .

The parameters of the interaction used for the plotting are given in Appendix 5. Note that, at $x=0$, we have a single system of species $b$, for which $T_{\mathrm{c}}^{(b)}=4.8028$. The case $x=1$ means the existence of a single system of species $a$, for which $T_{\mathrm{c}}^{(a)}=3.9502$.

The reduced form of Eq. (38) is

$\rho_{+}^{3}+p_{s} \rho_{+}+q_{s}=0$,

where

$p_{s}=-\frac{6 D_{a b}(x, T)}{a_{+}}, \quad q_{s}=-\frac{6 M_{a b}}{a_{+}}$.

For all $\tau>0$, Eq. (47) has a single solution

$\rho_{+}=\left(\frac{3}{a_{+}}\right)^{1 / 3}\left(A^{1 / 3}+B^{1 / 3}\right)$,

where

$$
\begin{aligned}
& A=M_{a b}+\sqrt{M_{a b}^{2}-M_{0 s} D_{a b}^{3}}, \\
& B=M_{a b}-\sqrt{M_{a b}^{2}-M_{0 s} D_{a b}^{3}} .
\end{aligned}
$$

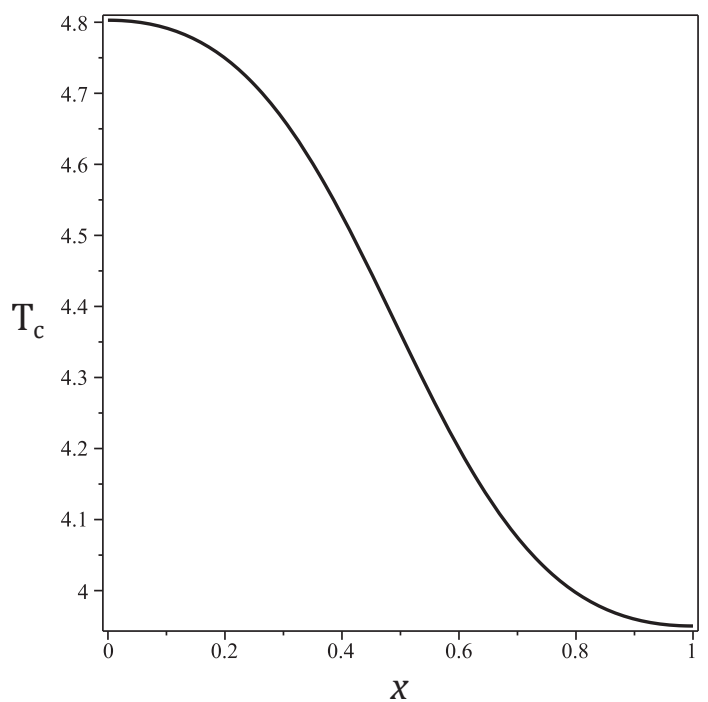

Fig. 1. Plot of the line of critical temperatures of the binary mixture

In these expressions,

$M_{0 s}=\frac{8}{9 a_{+}}$.

We find the temperature dependence of $D_{a b}$ from (35). Write an identity $D_{a b}=D_{a b}(x, T)-D_{a b}\left(x, T_{\mathrm{c}}\right)$, namely, according to (39), subtract zero from $D_{a b}$. Then

$D_{a b}=x^{2}\left(\frac{T_{\mathrm{c}}}{W_{a}\left(0, T_{\mathrm{c}}\right)}-\frac{T}{W_{a}(0)}\right)+$

$+(1-x)^{2}\left(\frac{T_{\mathrm{c}}}{W_{b}\left(0, T_{\mathrm{c}}\right)}-\frac{T}{W_{b}(0)}\right)$.

In view of the relation

$\tilde{a}_{2} W_{a}(0)=T_{c a}+2 \tilde{a}_{2} p_{a} T=$

$=\tilde{a}_{2} W_{a}\left(0, T_{\mathrm{c}}\right)+2 \tau \tilde{a}_{2} p_{a} T_{\mathrm{c}}$,

$\tilde{b}_{2} W_{b}(0)=T_{c b}+2 \tilde{b}_{2} p_{b} T=$

$=\tilde{b}_{2} W_{b}\left(0, T_{\mathrm{c}}\right)+2 \tau \tilde{b}_{2} p_{b} T_{\mathrm{c}}$,

we obtain $D_{a b}$ in a more compact form

$D_{a b}=x^{2} \tau \frac{W_{a}\left(0, T_{\mathrm{c}}\right)-2 p_{a}}{\beta_{\mathrm{c}} W_{a}\left(0, T_{\mathrm{c}}\right) \beta_{\mathrm{c}} W_{b}(0)}+$

$+(1-x)^{2} \tau \frac{W_{b}\left(0, T_{\mathrm{c}}\right)-2 p_{b}}{\beta_{\mathrm{c}} W_{b}\left(0, T_{\mathrm{c}}\right) \beta_{\mathrm{c}} W_{a}(0)}$.

The coefficient $D_{a b}$ is negative for all $\tau>0$ and positive at $T<T_{\mathrm{c}}$. It is proportional to $\tau$, like a similar coefficient in the case of a single system [25]. 


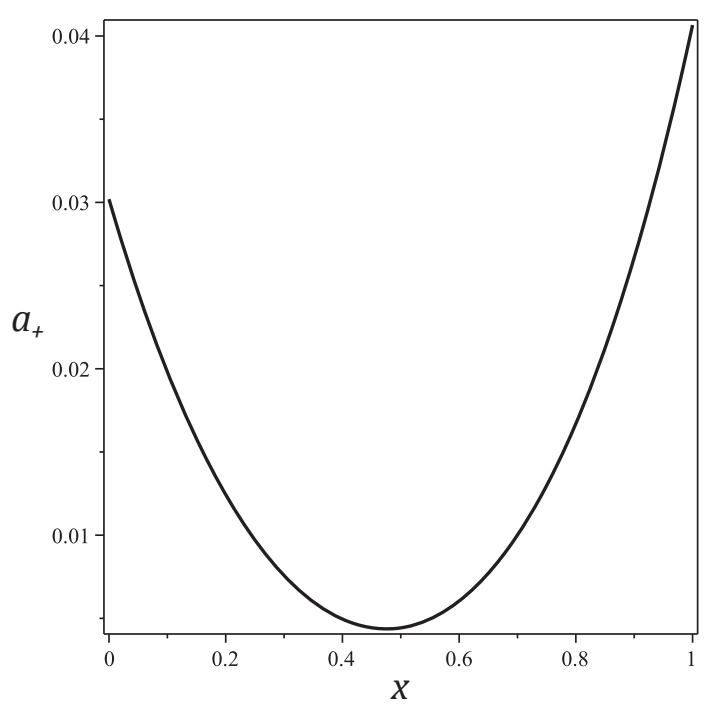

Fig. 2. Plot of the coefficient $a_{+}$given in (35) as a function of $x$

Solutions of Eq. (47) are similar to solutions of the analogous equation in the case of a single system (see [25]). The difference is that the critical temperature of the mixture depends on the mixing parameter $x$, as well as the coefficient $a_{+}=a_{+}(x)$. The latter case is shown in Fig. 2.

In the temperature range $T<T_{\mathrm{c}}$, Eq. (47) has three real solutions, which can be found with the use of the method suggested in work [25].

The solutions $\rho_{+}$are functions of the temperature $T$, the effective chemical potential $M_{a b}$, and the mixing parameter $x$. Therefore, it is possible to visualize the function $\rho_{+}\left(T, M_{a b}, x\right)$ only as separate projections on the surfaces:

$$
\begin{aligned}
& \left.\rho_{+}\right|_{T=\mathrm{const}}=\rho_{+}^{(1)}\left(M_{a b}, x\right), \\
& \left.\rho_{+}\right|_{x=\mathrm{const}}=\rho_{+}^{(2)}\left(M_{a b}, T\right), \\
& \left.\rho_{+}\right|_{M_{a b}=\mathrm{const}}=\rho_{+}^{(3)}(T, x) .
\end{aligned}
$$

Each of these projections reflects a particular physical process. The quantity $\rho_{+}^{(3)}$ is of importance, since it determines the Widom line of a binary mixture. It is easy to verify that, for all $T>T_{\mathrm{c}}$ at $M_{a b}=0$, we have $\rho_{+}\left(M_{a b}=0\right)=0$. Then we find the pressure, by using the well-known formula

$$
P V=k_{\mathrm{B}} T \ln \Xi \text {. }
$$

We obtain

$P v=k_{\mathrm{B}} T\left[\frac{1}{N_{v}} \ln \left(g_{v}^{(a)} g_{v}^{(b)}\right)+E_{W}^{(a)}+E_{W}^{(b)}\right]$,

where, according to (16), we have

$$
\begin{aligned}
& E_{W}^{(a)}=a_{0}-\frac{\beta}{2}\left(\mu_{a}^{(W)}\right)^{2}+m_{a}\left(a_{1}+\mu_{a}^{(W)}\right)+ \\
& +\frac{m_{a}^{2}}{2} d_{a}(0)+\frac{a_{3}^{4}}{8 a_{4}^{3}}, \\
& E_{W}^{(b)}=b_{0}-\frac{\beta}{2}\left(\mu_{b}^{(W)}\right)^{2}+m_{b}\left(b_{1}+\mu_{b}^{(W)}\right)+ \\
& +\frac{m_{b}^{2}}{2} d_{b}(0)+\frac{b_{3}^{4}}{8 b_{4}^{3}}
\end{aligned}
$$

moreover,

$$
\begin{aligned}
& \mu_{a}^{(W)}=-a_{1}-m_{a} d_{a}(0)+\frac{a_{3}^{3}}{a_{4}^{2}}, \\
& \mu_{b}^{(W)}=-b_{1}-m_{b} d_{b}(0)+\frac{b_{3}^{3}}{b_{4}^{2}} .
\end{aligned}
$$

The temperature dependence hides in the quantities $d_{\delta}(0)$. It is easy to see from the above formulas that the pressure along the Widom line is a function of the relative temperature $\tau$ and the parameter $x$. The dependence on $x$ eventuates from $d_{\delta}(0)$ containing the critical temperature $T_{\mathrm{c}}(x)$, which is a function of the mixing parameter:

$$
\begin{aligned}
& d_{a}(0)=-\tau \tilde{a}_{2} \frac{T_{\mathrm{c}}}{T_{\mathrm{c}}^{(a)}} \frac{1-\omega_{0 a}}{1+\omega_{0 a} \tau_{a}}- \\
& -\tilde{a}_{2} \frac{T_{\mathrm{c}}-T_{\mathrm{c}}^{(a)}}{T_{\mathrm{c}}^{(a)}} \frac{1-\omega_{0 a}}{1+\omega_{0 a} \tau_{a}} \\
& d_{b}(0)=-\tau \tilde{b}_{2} \frac{T_{\mathrm{c}}}{T_{\mathrm{c}}^{(b)}} \frac{1-\omega_{0 b}}{1+\omega_{0 b} \tau_{b}}- \\
& -\tilde{b}_{2} \frac{T_{\mathrm{c}}-T_{\mathrm{c}}^{(b)}}{T_{\mathrm{c}}^{(b)}} \frac{1-\omega_{0 b}}{1+\omega_{0 b} \tau_{b}}
\end{aligned}
$$

where $\tau_{a}$ and $\tau_{b}$ are given in (22). For $\omega_{\delta}$, we have

$\omega_{0 a}=2 \tilde{a}_{2}, \quad \omega_{0 b}=2 \tilde{b}_{2}$.

At $x=0$ and $x=1$,we get, respectively, the Widom lines of a single subsystem of species $b$ and $a$. For all $x \in(0,1)$, the set of Widom lines forms a surface represented in Fig. 3.

ISSN 2071-0194. Ukr. J. Phys. 2020. Vol. 65, No. 9 


\section{The Equation for Density}

The general principles of statistical mechanics give the equation for average numbers of particles of both species $\left\langle N_{a}\right\rangle$ and $\left\langle N_{b}\right\rangle$. Denote

$n_{a}=\frac{\left\langle N_{a}\right\rangle}{N_{v}}, \quad n_{b}=\frac{\left\langle N_{b}\right\rangle}{N_{v}}$.

Then, according to $[23,25]$, we have

$n_{a}=\frac{1}{N_{v}} \frac{\partial \ln \Xi}{\partial \beta \mu_{a}}, \quad n_{b}=\frac{1}{N_{v}} \frac{\partial \ln \Xi}{\partial \beta \mu_{b}}$.

With regard for $(28)$, we find

$$
\begin{aligned}
& n_{a}=-M_{a}+n_{\mathrm{c}}^{(a)}+\tilde{a}_{2} \gamma \tau_{a} \rho_{n}, \\
& n_{b}=-M_{b}+n_{\mathrm{c}}^{(b)}+\tilde{b}_{2} \gamma \tau_{b} \rho_{n} .
\end{aligned}
$$

The quantities $M_{a}, M_{b}$ are given in (30), which means that they are functions of $\tau$ and $\rho_{+}$. Equalities (30) yield

$x^{3} \rho_{n}^{3}+g_{a} x \rho_{n}+q_{a}=0$,

where

$g_{a}=\frac{6 \tilde{a}_{2}}{a_{4}}, \quad q_{a}=\frac{6\left(n_{\mathrm{c}}^{(a)}-n_{a}\right)}{a_{4}}$.

From (63), we have

$(1-x)^{3} \rho_{n}^{3}+g_{b}(1-x) \rho_{n}+q_{b}=0$,

where

$g_{b}=\frac{6 \tilde{b}_{2}}{b_{4}}, \quad q_{b}=\frac{6\left(n_{\mathrm{c}}^{(b)}-n_{b}\right)}{b_{4}}$.

Before writing the solutions of Eqs. (64) and (66), we introduce the total density of the mixture $n_{+}$and the concentration $\eta$ using the equations

$n_{a}=\eta n_{+}, \quad n_{b}=(1-\eta) n_{+}$.

Then

$n_{+}=n_{a}+n_{b}, \quad \eta=\frac{n_{a}}{n_{+}}, \quad 1-\eta=\frac{n_{b}}{n_{+}}$.

From (64), we have

$x \rho_{n}=-2 \bar{a} \cos \left(\frac{\alpha_{n}+\pi}{3}\right)$,

ISSN 2071-0194. Ukr. J. Phys. 2020. Vol. 65, No. 9

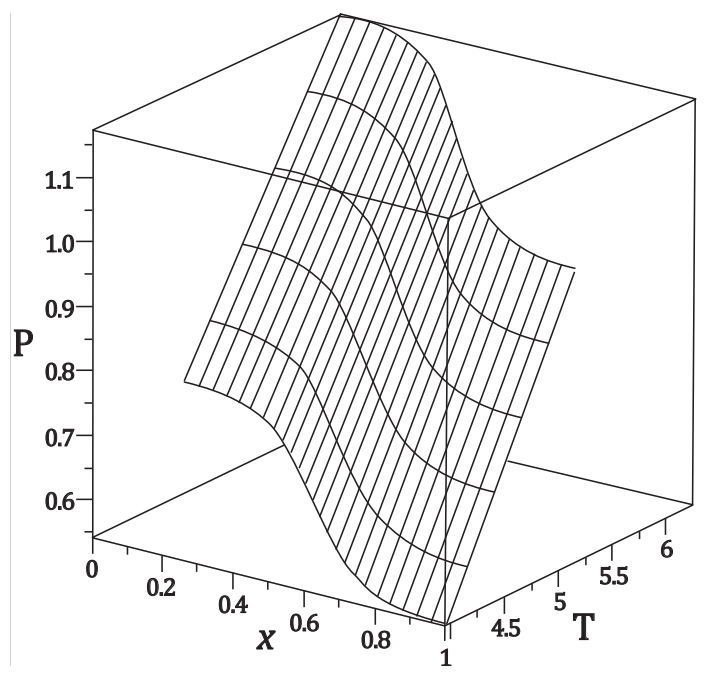

Fig. 3. Plot of the surface of the pressure extremum

where

$\alpha_{n}=\arccos \left(\frac{n_{\mathrm{c}}^{(a)}-\eta n_{+}}{n_{\mathrm{c}}^{(a)}}\right), \quad \bar{a}=\left(-\frac{2 \tilde{a}_{2}}{a_{4}}\right)^{1 / 2}$.

From (66), we have

$(1-x) \rho_{n}=-2 \bar{b} \cos \left(\frac{\beta_{n}+\pi}{3}\right)$

where

$\beta_{n}=\arccos \left(\frac{n_{\mathrm{c}}^{(b)}-(1-\eta) n_{+}}{n_{\mathrm{c}}^{(b)}}\right), \quad \bar{b}=\left(-\frac{2 \tilde{b}_{2}}{b_{4}}\right)^{1 / 2}$.

The sum of expressions (70) and (72) equals

$\rho_{n}=-2 \bar{a} \cos \left(\frac{\alpha_{n}+\pi}{3}\right)-2 \bar{b} \cos \left(\frac{\beta_{n}+\pi}{3}\right)$.

Note that $\rho_{n}$ fails to be a function of the temperature. It has a restricted range, since $\bar{a}$ and $\bar{b}$ are finite quantities. The subtraction of (70) and (72) gives the equation

$x=\frac{1}{2}\left[1+\frac{\bar{a} \cos \left(\frac{\alpha_{n}+\pi}{3}\right)-\bar{b} \cos \left(\frac{\beta_{n}+\pi}{3}\right)}{\bar{a} \cos \left(\frac{\alpha_{n}+\pi}{3}\right)+\bar{b} \cos \left(\frac{\beta_{n}+\pi}{3}\right)}\right]$,

which allows us to find the relation between the mixing parameter $x$ and the concentration $\eta$ for an arbitrary value of the total density $n_{+}$. 


\section{Conclusions}

In this article, we represent the investigation of the behavior of a binary asymmetric mixture at $T>T_{\mathrm{c}}$. Among the results is a two-dimensional ana$\log$ of the Widom line for different values of the mixing parameter $x \in[0,1]$. The equation of state of the system, which includes the dependence on the parameter $x$, is calculated. This parameter links to the concentration and is also used to determine the critical temperature of the mixture. In the boundary cases $x=0$ and $x=1$, the formulas obtained here describe separately the subsystems of particles of species $b$ and $a$, respectively. In the case of $x \in(0,1)$, we have a mixture of components, that is characterized by the critical temperature $T_{\mathrm{c}}$ (see Fig. 1). The Widom line shifts on the surface shown in Fig. 3, as the composition of the mixture changes.

\section{APPENDIX A}

\section{Parameters of the Model}

The following values of the interaction potential parameters are used for numerical calculations and plots.

For both species, $\alpha_{a}=\alpha_{b}=\alpha$, as well as $R_{0}^{(a)}=R_{0}^{(b)}=R_{0}$ :

$R_{0}=5.3678, \quad \alpha=1.8167, \quad \alpha_{R}=0.3385$,

which coincide with the parameters of the Morse potential for sodium $[29,30]$. We choose the following values of the parameters $\gamma_{a}$ and $n_{0}^{(a)}$ of the potential $U_{a}(r)$ (see $(1)$ ) corresponding to species $a$ :

$\gamma_{a}=1.6500, \quad n_{0}^{(a)}=1.7255$.

The values (A2) give

$p_{a}=1, \quad \alpha_{a}^{*}=5$,

see (12). This set of parameters eventuates in the following values of the coefficients $a_{n}$ (see (11) and (18)):

$a_{0}=1.1220, \quad a_{1}=0.7506, \quad a_{2}=0.3413$,

$a_{3}=0.0265, \quad a_{4}=-0.0407, \quad \tilde{a}_{2}=0.3500$.

The values of the parameters $\gamma_{b}$ and $n_{0}^{(b)}$ of the potential $U_{b}(r)$ differ from $\gamma_{a}$ and $n_{0}^{(a)}$ :

$\gamma_{b}=1.6000, \quad n_{0}^{(b)}=1.7613$.

They give

$p_{b}=1, \quad \alpha_{b}^{*}=5.5$

and the following values of the coefficients $b_{n}$ :

$b_{0}=1.1951, \quad b_{1}=0.7832, \quad b_{2}=0.3437$,

$b_{3}=0.0231, \quad b_{4}=-0.0302, \quad \tilde{b}_{2}=0.3526$.
The normalization constants given in (2) equal

$C_{H}^{(a)}=1.2544, \quad A_{a}=0.2217$,

$C_{H}^{(b)}=1.2938, \quad A_{b}=0.2742$.

The values of the critical temperatures of separate single systems of species $a$ and species $b$ are

$k_{\mathrm{B}} T_{\mathrm{c}}^{(a)}=3.9502, \quad k_{\mathrm{B}} T_{\mathrm{c}}^{(b)}=4.8028$.

1. A. Oleinikova, L. Bulavin, V. Pipich. Critical anomaly of shear viscosity in a mixture with an ionic impurity. Chem. Phys. Let. 278, 121 (1997).

2. V.I. Petrenko, M.V. Avdeev, L.A. Bulavin, P. Kopcansky. Impact of polyethylene glycol on aqueous micellar solutions of sodium oleate studied by small-angle neutron scattering, Colloids and Surfaces A: Physicochemical and Engineering Aspects 480, 191 (2015).

3. M. Isaiev, S. Burian, L. Bulavin, M. Gradeck, F. Lemoine, K. Termentzidis. Efficient tuning of potential parameters for liquid-solid interactions. Molecular Simulation 42, 910 (2016).

4. P.H. Van Konynenberg, R.L. Scott. Critical lines and phase equilibria in binary van der Waals mixtures. Phil. Trans. Royal Soc. of London. Series A 298, 495 (1980).

5. Y. Levin, M.E. Fisher. Criticality in the hard-sphere ionic fluid. Physica A 225, 164 (1996).

6. A. Parola, L. Reatto. Liquid state theories and critical phenomena. Advances in Phys. 44, 221 (1995).

7. O.V. Patsagan, I.R. Yukhnovskii. Functional of the grand partition function in the method of collective variables with distinguished reference system. Multicomponent system. Theor. Math. Phys. 83, 387 (1990).

8. O.V. Patsahan. On the microscopic theory of phase transitions in binary fluid mixtures. Physica A 272, 358 (1999).

9. I.R. Yukhnovskii, M.F. Holovko. Statistical theory of classical equilibrium systems (Naukova dumka, 1980) (in Russian).

10. O. Patsahan, I. Mryglod. Functional representation of the grand partition function of a multicomponent system of charged particles. Condens. Matter Phys. 9, 659 (2006).

11. M.P. Kozlovskii, O.V. Patsahan, R.S. Melnyk. A study of the gas-liquid critical point of a binary symmetric mixture. Ukr. J. Phys. 45, 381 (2000).

12. M.P. Kozlovskii, O.V. Patsahan, R.S. Melnyk. Thermodynamic characteristics of binary symmetric mixture in the vicinity of the vapor-liquid critical point. Ukr. J. Phys. 49, 55 (2004).

13. J.D. Bernal. A geometrical approach to the structure of liquids. Nature 183, 141 (1959).

14. J.M. Stubbs. Molecular simulations of supercritical fluid systems. J. Supercrit. Fluid 108, 104 (2016).

15. T.J. Yoon, Y.-W. Lee. Current theoretical opinions and perspectives on the fundamental description of supercritical fluids. J. Supercrit. Fluid 134, 21 (2018).

ISSN 2071-0194. Ukr. J. Phys. 2020. Vol. 65, No. 9 
16. P.F. McMillan, H.E. Stanley. Going supercritical. Nature Physics 6, 479 (2010).

17. Ž. Knez, E. Markočič, M. Leitgeb, M. Primožič, M.K. Hrnčič, M. Škerget. Industrial applications of supercritical fluids: A review. Energy 77, 235 (2014).

18. G. Brunner. Applications of Supercritical Fluids. Annu. Rev. Chem. Biomol. Eng. 1, 321 (2010).

19. B. Widom. Equation of state in the neighborhood of the critical point J. Chem. Phys. 43, 3898 (1965).

20. D.T. Banuti. Crossing the Widom-line-supercritical pseudo-boiling. J. Supercrit. Fluid 98, 12 (2015).

21. G.G.Simeoni, T. Bryk, F.A. Gorelli, M. Krisch, G. Ruocco, M. Santoro, T. Scopigno. The Widom line as the crossover between liquid-like and gas-like behaviour in supercritical fluids. Nature Physics 6, 503 (2010).

22. M. Raju, D.T. Banuti, P.C Ma, M. Ihme. Widom lines in binary mixtures of supercritical fluids. Sci. Rep. 7, 3027 (2017).

23. M.P. Kozlovskii, O.A. Dobush. Phase transition in a cell fluid model. Condens. Matter Phys. 20, 23501 (2017).

24. M. Kozlovskii, O. Dobush. Representation of the grand partition function of the cell model: The state equation in the mean-field approximation. J. Mol. Liq. 215, 58 (2016).

25. M.P. Kozlovskii, O.A. Dobush. arXiv:1912.00769, (2019).

26. Y. Kozitsky, M. Kozlovskii, O. Dobush. Phase transitions in a continuum Curie-Weiss system: A quantitative analysis. In: Modern Problems of Molecular Physics (Springer, 2018), p. 229.

27. I.R. Yukhnovskii, M.P. Kozlovskii, I.V. Pylyuk. Thermodynamics of three-dimensional Ising-like systems in the higher non-Gaussian approximation: Calculational method and dependence on microscopic parameters. Phys. Rev. B 66, 134410 (2002).

28. M.V. Fedoryuk. Asymptotic methods in analysis. In: Analysis I: Integral Representations and Asymptotic Methods.
Edited by M.A. Evgrafov, R.V. Gamkrelidze (Springer, 1989).

29. R.C. Lincoln, K.M. Koliwad, P.B. Ghate. Morse-potential evaluation of second-and third-order elastic constants of some cubic metals. Phys. Rev. 157, 463 (1967).

30. J.K. Singh, J. Adhikari, S.K. Kwak. Vapor-liquid phase coexistence curves for Morse fluids. Fluid Phase Equilibria 248, 1 (2006)

Received 31.03.20

М.П. КозловсъКий, О.А. Добуш

ПОВЕДІНКА БІНАРНОї

АСИМЕТРИЧНОЇ СУМІШ ВЗАЄМОДІЮЧИХ

ЧАСТИНОК В НАДКРИТИЧНІЙ ОБЛАСТІ

Р е $з$ ю м е

Запропоновано метод опису фазової поведінки системи, яка складається із частинок двох сортів. Взаємодія кожного iз сортів описується властивим йому потенціалом взаємодії, що містить відштовхувальну та притягальну частини. Асиметричність забезпечується різними значеннями параметрів потенціалів взаємодії кожного із сортів. Проведено розрахунок великої статистичної суми бінарної системи в наближенні нульової моди. Для конкретних значень параметрів потенціалу взаємодії розраховано лінію критичних точок, які відповідають різним пропорціям компонент. Отримано рівняння, яке пов'язує введений нами параметр змішування $x$ із концентрацією системи. Знайдено явний вигляд виразу для тиску бінарної системи як функції відносної температури та параметра змішування $x$ для побудови лінії Відома. Встановлено, що для граничних значень цього параметра ( $x=0$ та $x=1$ ) рівняння стану суміші переходить у рівняння стану однієї з окремих компонент. 